\title{
Apical Extrusion of Intracanal Debris Using Two Engine Driven and Step-Back Instrumentation Techniques: An In-Vitro Study
}

\author{
Alper Kustarcia \\ Neslihan Akdemira \\ Seyda Herguner Sisob \\ Demet Altunbas ${ }^{a}$
}

\begin{abstract}
Objectives: The purpose of this study was to compare in-vitro the amount of debris extruded apically from extracted teeth, using K3, Protaper rotary instruments and manual step-back technique.

Methods: Forty five human single-rooted mandibular premolar teeth were randomly divided into 3 groups. The teeth in 3 groups were instrumented until reaching the working length with K3, Protaper rotary instruments and K-type stainless steel instruments with manual step-back technique, respectively. Debris extruded from the apical foramen was collected into centrifuge tubes and the amount was determined. The data obtained were analyzed using Kruskal-Wallis one-way analysis of variance and Mann-Whitney $U$ tests, with $P=.05$ as the level for statistical significance.

Results: Statistically significant difference was observed between K3, Protaper and step-back groups in terms of debris extrusion $(\mathrm{P}<$.05). Step-back group had the highest mean debris weight, which was significantly different from the $\mathrm{K} 3$ and Protaper groups $(\mathrm{P}<.05)$. The lowest mean debris weight was related to K3 group, which was significantly different from the Protaper group $(P<.05)$.

Conclusions: Based on the results, all instrumentation techniques produced debris extrusion. The engine-driven Ni-Ti systems extruded significantly less apical debris than step-back technique. However, Protaper rotary instruments extruded significantly more debris than K3 rotary instruments. (Eur J Dent 2008;2:233-239)
\end{abstract}

Key words: Protaper; K3; Engine-driven; Extruded debris; Positive rake angle; Negative rake angle.

\footnotetext{
- a Cumhuriyet University, Faculty of Dentistry, Department of Endodontics, Sivas, Turkey.

b Cumhuriyet University, Faculty of Dentistry, Department of Restorative Dentistry, Sivas, Turkey.

- Corresponding author: Alper Kustarci, Cumhuriyet University, Faculty of Dentistry, Department of Endodontics, Sivas, Turkey. Phone: +90 346 2191010/2764

Fax: +90346 2191237

E-mail: akustarciahotmail.com
}

\section{INTRODUCTION}

The cleaning of dentin within the root canal and the removal of inflamed and/or necrotic tissue remains as one of the most important steps in endodontic theraphy. ${ }^{1}$ Dentine chips, pulp tissue fragments, necrotic tissue, microorganisms and intracanal irrigants may be extruded from the apical foramen during the canal instrumentation. This is of concern since material extruded from 
the apical foramen may be related to postinstrumentation pain or to a flare-up. ${ }^{2}$

The inter-appointment flare-up is a true complication characterized by the development of pain, swelling or both, which commences within a few hours or days after root canal procedures and is of sufficient severity to require an unscheduled visit for emergency treatment. ${ }^{3}$ The causative factors of inter-appointment flare-ups comprise mechanical, chemical and/or microbial injury to the pulp or periradicular tissues. ${ }^{2,4}$ Apical extrusion of infected debris to the periradicular tissues is possibly one of the principal causes of postoperative pain. ${ }^{2,5}$ In asymptomatic chronic periradicular lesions associated with infected teeth, there is a balance between microbial aggression and host defense in the periradicular tissues. During chemo-mechanical preparation, if the microorganisms are apically extruded, the host will face a situation in which it will be challenged by a larger number of irritants than it before. Consequently, there will be a transient disruption in the balance between aggression and defense in such a way that the host will mobilize an acute inflammation to re-establish the equilibrium. ${ }^{3}$

All preparation techniques and instruments have been reported to be associated with extrusion of infected debris, even when preparation is maintained short of the apical terminus. ${ }^{6-9}$ Vande Visse and Brilliant ${ }^{6}$ first quantified the amount of debris apically extruded during instrumentation. They found that instrumentation with irrigant produced extrusion, whereas instrumentation without irrigant produced no collectible debris. Martin and Cunningham ${ }^{10}$ reported that less debris was extruded when the intracanal preparation was accomplished with an ultrasonic instrument. Reddy and Hicks ${ }^{7}$ compared apical debris extrusion between hand and engine-driven $\mathrm{Ni}$-Ti instruments (Lightspeed and Profile Series 29), comparing the mean weights of apically extruded debris, showed that step-back instrumentation produced significantly more debris than the two engine-driven $\mathrm{Ni}$-Ti techniques.

During the last decade root canal preparation with engine-driven $\mathrm{Ni}$-Ti instruments has become popular. More recently advanced instrument designs including non-cutting tips, radial lands, different cross sections and varying tapers have been to improve working safety, to shorten time, and to create a greater flare of preparations. ${ }^{11}$

The K3 rotary instrument (SybronEndo, West Collins, California, USAl is reported to have a slightly positive rake angle in combination with so-called radial land relief and asymmetrical cross-sectional design. ${ }^{11}$ The peripheral blade relief areas are alleged to have two functions: (i) to increase the peripheral mass in order to increase the instruments resistance to fracture and (ii) to reduce the amount of area of the radial lands that comes in contact with the canal wall in order to reduce frictional resistance. ${ }^{12}$

In the progressive ProTaper system (Dentsply Maillefer, Ballaigues, Switzerland), the shaping files (S) have an increasing taper from tip to coronal, whereas the finishing files ( $F$ ) have a decreasing taper. It has been claimed that the increasing taper instruments have enhanced flexibility in the middle region and at the tip, and that the decreasing taper instruments provide a larger taper in the important apical region but make them stiff. ${ }^{13}$ Also ProTaper rotary instruments have a convex triangular cross-sectional design, a noncutting safety tip and a flute design that combines multiple tapers within the shaft. ${ }^{14}$

The purpose of this study was to compare in vitro the amount of debris extruded apically from extracted teeth, using K3, Protaper rotary instruments and manual step-back technique.

\section{MATERIALS AND METHODS}

Selection and preparation of teeth

In this study, forty five freshly extracted mandibular premolar teeth were used. All teeth were analyzed with digital radiographs (Schick Tech. Inc., Long Island City, NY, USAl in buccal and proximal directions to check for a single canal. Teeth with calcification and open apices were excluded and one apical foramen and mature apices, curvature between 0-10 degrees were selected. ${ }^{15}$ The teeth were cleaned of debris and soft tissue remnants and were stored in physiological saline solution $(\mathrm{NaCl})$ at $+4^{\circ} \mathrm{C}$ until required.

The buccal cusp edge of each tooth was then flattened as a reference point, coronal access was prepared conventionally with a high-speed bur and the canal was broached the remove the bulk of the soft tissue. A size 15 file was extended just beyond the apical foramen to ensure that the 
root canals were patent before instrumentation. No other files were passed out the apical foramen again. The teeth were randomly divided into three experimental groups comprising 15 teeth each.

\section{Test apparatus}

The experimental model system used to evaluate debris extrusion is presented in (Figure 1). Holes were created in the stoppers of centrifuge tubes and teeth were inserted under pressure through the stoppers, which were fixed to the cementoenamel junction by means cyanoacrylate. A bent 27-gauge needle was also forced alongside the stopper to use as a drainage cannula, balance between the air pressure inside and outside the centrifuge tubes and an electrode for the electronic working length determination during canal instrumentation. Then centrifuge tubes were fitted into the vials.

Centrifuge tubes were entirely filled with $0.9 \%$ $\mathrm{NaCl}$ and the tooth-stopper-needle unit was fitted into the mouth of the centrifuge tube. Then some wax was placed to junction of stopper unit and centrifuge tube for prevent liquid leakage.

\section{Root canal preparation}

Root canal preparation and working length measurement completed using Endomaster (EMS, SA, Switzerland) endodontic hand piece at lowspeed (300 rpm) and automatic reverse function mode. A lip clip was attached to the needle. Rotary Ni-Ti file was placed into the root canal and advanced apically until 1 LED was read on the console of the Endomaster. Total volume $9 \mathrm{ml}$ of $0.9 \% \mathrm{NaCl}$ was used for irrigating root canals for each tooth. The 27-gauge needle tip inserted passively and never

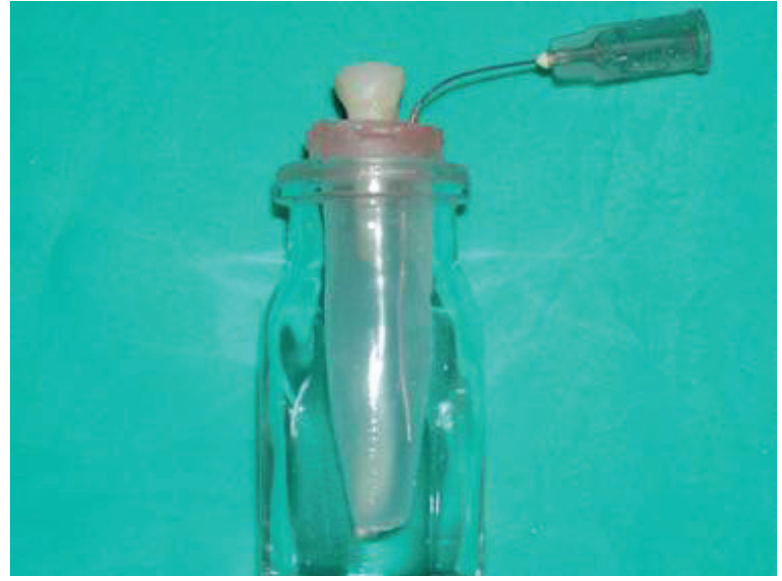

Figure 1. The experimental model system. allowed to bind as the irrigant was being deposited into the canal.

The series used for each instrumentation technique was as follows:

Group 1 (K3 Group): K3 rotary instruments were used in a crown-down technique and advanced apically in a gentle pecking motion until the first sign of resistance was felt. File sequences used were: size $.06 / 30$ was used $1 / 3$ of the working length, size $.06 / 25$ was used $1 / 2$ of the working length, size $.06 / 20$ was used between $1 / 2$ and $2 / 3$ of the working length, instruments of size .04/20, $.04 / 25, .04 / 30$ were used to the working length. In order to prevent blockage of the apical foramen, size $15 \mathrm{~K}$-file was advanced to the full working length between each file.

Group 2 (Protaper Group): Protaper rotary instruments were used in a crown-down manner according to the manufacturer's instructions using a gentle in and out motion. Instruments were withdrawn when resistance was felt and changed for the next instrument. File sequences used were: Sx files were used until resistance was encountered (4-5 $\mathrm{mm}$ from the working length), S1 and S2 files were used 2/3 of the working length and F1,F2,F3 files were used of the working length. Size $15 \mathrm{~K}$-file was used at the working length between each file in order to prevent apical blockage.

Group 3 (Step-back Group): K-file instruments were used in a step-back manner and preparation was performed with rotational forces. K-files were used first with a quarter clockwise rotation followed by a pull-back motion and used repeatedly until reaching the working length. Apical preparation

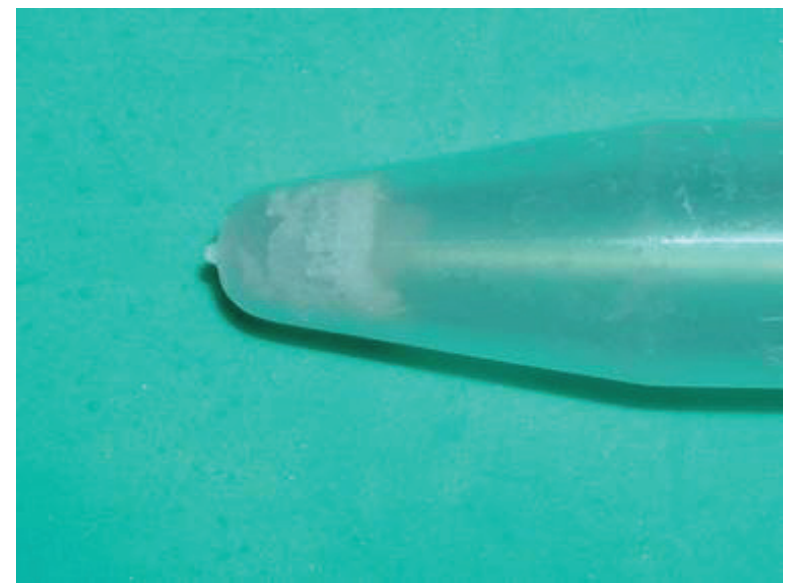

Figure 2. Appearance of salt deposit and dry debris in a centrifuge tube. 
was continued up to size 30 and after completion of this stage the step-back technique was used with a reduction of $1 \mathrm{~mm}$ for each file until size 45 and size $15 \mathrm{~K}$-file was used at the working length between each file after size 25 file in order to prevent apical blockage.

Following this procedure, tooth-stopper-needle unit were removed along the collecting tubes and centrifuge tubes were stored to evaporate the fluid $37^{\circ} \mathrm{C}$ for 21 days and after this procedure, salt and debris collected (Figure 2). Then centrifuge tubes were weighed by means of an analytic balance at 10-5 gram precision. Following this procedure centrifuge tubes were cleaned with distilled water, dried and filled with $0.9 \% \mathrm{NaCl}$. Tooth-stopperneedle unit fitted into $\mathrm{NaCl}$ filled centrifuge tubes, excess of the $\mathrm{NaCl}$ drained out through the needle and then stopper unit was removed, and $37^{\circ} \mathrm{C}$ for 21 days centrifuge tubes were stored to evaporate the fluid again. Salt deposits and tubes were weighted to $10^{-5}$ precision. Mean first weights were compared with the mean second weights and the difference was recorded as the weight of the extruded debris.

\section{Statistical analysis}

Statistical tests were performed using SPSS (Version 9.0, SPSS Inc., Chicago, IIl, USA). Data were analyzed statistically using Kruskal-Wallis one-way analysis of variance and Mann-Whitney $U$ tests. The level of statistical significance was set at $\mathrm{P}=.05$.

\section{RESULTS}

The mean weight and standard deviation for each instrumentation group are presented in Table 1. The results indicated that all instruments tested caused a measurable apical extrusion of debris. Statistically significant difference was observed between K3, Protaper and step-back groups in terms of debris extrusion ( $P<.05)$. Step-back group had the highest mean debris weight, which was significantly different from the $\mathrm{K} 3$ and Protaper groups $(P<.05)$. The lowest mean debris weight was related to K3 group, which was significantly different from the Protaper group $(P<.05)$.

\section{DISCUSSION}

The main objective of the present study was to assess the apical extrusion of the debris as a result of canal shaping by different $\mathrm{Ni}$-Ti files and step-back technique.

In this study, method used for debris collection was a modification of the technique used by Ferraz et $\mathrm{al}^{8}$ for debris collection. In that technique, the teeth were forced through a hole in rubber stopper, then this unit was fitted into the mouth of the vial and the apical part of the root was suspended within the centrifuge tube, which acted as a collecting container for apical debris. Also the debris adhering to the root surface were collected by washing off the apex with $1 \mathrm{ml}$ distilled water into the centrifuge tube after preparation. However, the teeth were directly in the centrifuge tubes filled with $0.9 \% \mathrm{NaCl}$ as an electrolyte to ensure the functioning of the apex locator and these units were fitted in vials in our technique. The 27-gauge needle that functioned as an air vent in previous debris extrusion studies functioned as a drain to reflect the amount of the extruded irrigant.

It must be emphasized that the results of this study should not be directly extrapolated to the clinical situation. No attempt has been made to simulate the presence of vital pulp or periapical tissues, an in vivo model may give different result, as the periapical tissues may serve as a natural barrier, inhibiting debris extrusion. If the quantities of debris extruded in this study were extruded routinely in clinical practice, a higher incidence of postoperative pain might be anticipated. Results may also differ because of positive and negative pressure at the apex and with normal or pathological periapical tissues. Furthermore, this study was limited to teeth with

Table 1. The mean weight of extruded debris.

\begin{tabular}{lccc}
\hline Instrumentation technique & Total (n) & Mean weight (mg) & Std. deviation \\
\hline K3 $^{\text {a }}$ & 15 & 0.022 & 0.013 \\
Protaper $^{\mathrm{b}}$ & 15 & 0.038 & 0.021 \\
Step-back $^{c}$ & 15 & 0.051 & 0.011 \\
\hline
\end{tabular}

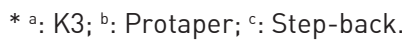


mature root morphology. The observed results should not be generalized to teeth with immature root development and open apicies.

Apical extrusion of irrigants and debris has been studied extensively because of its clinical relevance, particularly since it may cause flareups, originated by the introduction of bacteria, pulpal tissue and irrigating solutions into the periapical tissues. ${ }^{2}$ Many factors affect the amount of extruded intracanal materials such as; instrumentation technique, instrument type, instrument size and preparation endpoint and irrigation solution. $6,8,9,16-19$

Irrigation is a necessary and important phase of cleansing the canal. The irrigant functions as a lavage and flush, a solvent, a disinfectant, and a lubricant within the canal. ${ }^{10}$ Many liquids have been used as canal irrigants, for example sterile water, $\mathrm{NaCl}$, local anesthetic solution, sodium hypochlorite solution and hydrogen peroxide solution. ${ }^{20}$ According to Abou-Rass and Piccino, ${ }^{21}$ deep delivery of the irrigation solution into root canals results in more effective removal debris. The disadvantage of this method of delivery may be an increased apical extrusion. Vande Visse and Brilliant $^{6}$ showed that the introduction of fluid into the canal made instrumentation easier, but fluid also permitted debris to pass more easily out the apical foramen. In the present study, total volume $9 \mathrm{ml}$ of $0.9 \% \mathrm{NaCl}$ was used for irrigating root canals between each files. The 27-gauge needle tip inserted passively and never allowed to bind as the irrigant was being deposited into the canal. Same irrigation procedure was applied to all teeth, in this way debris extrusion effect of the $\mathrm{Ni}-\mathrm{Ti}$ instruments was investigated.

Myers and Montgomery ${ }^{22}$ clearly showed that a working length $1 \mathrm{~mm}$ short of canal length contributed to significantly less debris extrusion. Beeson et $\mathrm{al}^{18}$ reported that, when the instrumentation was performed to the apical foramen, significantly more debris was forced apically than when instrumentation was $1 \mathrm{~mm}$ short. In the present study, the canal working length was $1 \mathrm{~mm}$ short of the apical foramen and working length measurements were completed with the Endomaster electronic apex-locating handpiece with "auto-reverse function mode".

Formation of an apical plug is unpredictable, and for reasons of standardization, in order to prevent blockage of the apical foramen, size 15 K-file was used at the full working length between each file. If apical plugs were formed, it is likely that the amount of debris extruded would have been less than observed in this study.

The extrusion produced by the various techniques was expected, because it is considered a problem of all canal instrumentation methods. ${ }^{6}$ However, preparation techniques effect the volume of the extruded debris. In a study, Zarrabi et $\mathrm{al}^{19}$ compared Profile, RaCe and FlexMaster rotary instruments with step-back technique and reported that all instrumentation techniques extruded debris. However, step-back technique extruded greater debris than rotary instruments. Ferraz et $\mathrm{al}^{8}$ noted that preparation with Profile rotary instruments extruded less debris. In a study, Azar and Ebrahimi ${ }^{23}$ showed that all instrumentation techniques produced extruded debris and irrigant. The amount extruded debris was lower in the Profile and Protaper rotary systems than manual step-back technique.

In the present study, using engine-driven nickel-titanium instruments for the crown-down technique extruded less debris than K-files for the step-back technique. Early flaring of the coronal part of the preparation may improve instrument control during preparation of the apical third of the canal, and also because of the rotary motion, which tends to direct debris toward the orifice, avoiding its compactation in the root canal. In the step-back technique, the reason for more apical extrusion of debris is that the file acting in the apical one third acts as a piston that tends to push the debris through the foramen and less space is available to flush it out coronally.

Although a negative rake angle is least aggressive, the cutting efficiency of a file can also be affected by the blank design. For example, the ProTaper rotary instrument has a negative rake angle but due to its modified $\mathrm{K}$ blade and progressive taper in combination with the sharp cutting edges, the instrument cuts very effectively. ${ }^{24}$ In the present study, Protaper rotary instrument extruded significantly more debris than K3 rotary instrument. The final file of the ProTaper rotary instrument $\mathrm{F} 3$ has an apical taper of 0.09 , which is much larger than the $\mathrm{K} 3$ that has a 0.04 taper. The large taper of the F3 instrument increases the stiffness of the tip and the use of larger and greater 
taper apical files performed more aggressive cutting in root canals and this could be a cause of the more apically extruded debris by Protaper rotary instrument.

However, K3 rotary instrument is reported to have a slightly positive rake angle in combination with a radial land relief. ${ }^{11} \mathrm{~A}$ positive rake angle tends to increase the cutting efficiency of the file. ${ }^{11,25}$ Also Walsch ${ }^{26}$ reported that files with a positive rake along with a variable helical flute angle enabled better dentine cutting and debris removal from the canal system. Dentine chips resulting from the $\mathrm{K} 3$ rotary instrument cutting action are easily dislodged from the working area and carried to the orifice via its unique helical angle. ${ }^{27}$ In the present study, removal debris was carried to orifice of root canal better and less debris extruded in the $\mathrm{K} 3$ group due to the $\mathrm{K} 3$ rotary instruments have positive rake angle and variable helical flute design.

\section{CONCLUSIONS}

Based on the results, all instrumentation techniques produced debris extrusion. The enginedriven $\mathrm{Ni}$-Ti systems extruded significantly less apical debris than step-back technique. However, Protaper rotary instruments extruded significantly more debris than $\mathrm{K} 3$ rotary instruments.

\section{REFERENCES}

1. Stewart GG. The importance of chemomechanical preparation of the root canal. Oral Surg Oral Med Oral Pathol 1955;8:993-997.

2. Seltzer S, Naidorf IJ. Flare-ups in endodontics: I. Etiological factors. J Endod 1985;11:472-478.

3. Siqueira JF Jr. Microbial causes of endodontic flare-ups. Int Endod J 2003;36:453-63.

4. Torabinejad M, Kettering JD, McGraw JC, Cummings RR, Dwyer TG, Tobias TS. Factors associated with endodontic interappointment emergencies of teeth with necrotic pulps. J Endod 1988;14:261-266.

5. Wittgow WC Jr, Sabiston CB Jr. Microorganisms from pulpal chambers of intact teeth with necrotic pulps. $J$ Endod 1975;1:168-171.

6. Vande Visse IE, Brillant JD. Effect of irrigation on the production of extruded material at the root apex during ilıstrumentation. J Endod 1975; 1:243-246.

7. Martin H, Cunningham WT. The eftect of endosonic and hand manuplation on the amount of root canal material extruded. Oral Surg Oral Med Oral Pathol 1982;53:611-613.
8. Ferraz CCR, Gomes NV, Gomes BPFA, Zaia AA, Teixeria FB, Souza-Fjlho FJ. Apical extrusion of debris and irrigants using two hand and three engine-driven instrumentation techniques. Int Endod J 2001;34:354-358.

9. Salzgeber RM, Brilliant JD. An in vivo evaluation of the penetration of an irrigating solution in root canals. $J$ Endod 1977;3:394-398.

10. Reddy SA, Hicks ML. Apical extrusion of debris using two hand and two rotary instrumentation techniques. $J$ Endod 1998;24:180-183.

11. Bergmans L, Van Cleynenbreugel J, Wevers M, Lambrechts P. Mechanical root canal preparation with NiTi rotary instruments: Rationale, performance and safety. Status report for the American Journal of Dentistry. Am J Dent 2001;14:324-333.

12. Schäfer $E$, Florek H. Efficiency of rotary nickel-titanium K3 instruments compared with stainless steel hand K-Flexofile. Part 1. Shaping ability in simulated curved canals. Int Endod J 2003;36:199-207.

13. Bergmans L, Van Cleynenbreugel J, Beullens $M$, Wevers M, Van Meerbeek B, Lambrechts P. Progressive versus constant tapered shaft design using $\mathrm{Ni}-\mathrm{Ti}$ rotary instruments. Int Endod J 2003;36:288-295.

14. Guelzow A, Stamm O, Martus P, Kielbassa AM Comparative study of six rotary nickel-titanium systems and hand instrumentation for root canal preparation. Int Endod $J$ 2005; 38:743-752.

15. Schneider SW. A comparison of canal preparations in straight and curved root canals. Oral Surg Oral Med Oral Pathol 1971;32:271-275.

16. Tinaz AC, Alacam T, Uzun O, Maden M, Kayaoglu G. The effect of disruption of apical constriction on periapical extrusion. J Endod 2005;31:533-535.

17. Fairburn DR, McWalter GM, Montgomery S, The effect of four preparation techniques on the amount of apically extruded debris. J Endod 1987;13:102-108.

18. Beeson T, Hartwell G, Thornton J, Gunsolley J, Comparison of debris extruded apically in straight canals: conventional filling versus Profile 04 Taper series 29. JEndod 1998;24:1822.

19. Zarrabi MH, Bidar M, Jafarzadeh H. An in vitro comparative study of apically extruded debris resulting from conventional and three rotary (Profile, Race, FlexMaster) instrumentation techniques. J Oral Sci 2006;48:85-88.

20. Saunders EM, Saunders WP. Conventional root canal therapy. I Preparation of the root canal system. In: Harty Fj, ed. Endodontics in Clinical Practice, 3rd edn. London, UK: Wright 1990;128-161. 
21. Abou-Rass M, Piccinino M. The effectiveness of four clinical irrigation methods on the removal of root canal debris. Oral Surg Oral Med Oral Pathol 1982;54:323-328.

22. Myers GL, Montgomery S. A comparison of weights of debris extruded apically by conventional filing and Canal Master techniques. J Endod 1991;17:275-279.

23. Azar NG, Ebrahimi G, Apically-extruded debris using the ProTaper system. Aust Endod J 2005;31:21-23.

24. Koch KA, Brave DG. Real World Endo: Design features of rotary files and how they affect clinical performance. Oral Health 2002:1:39-49.

25. Wildey WL, Senia ES, Montgomery S. Another look at root canal instrumentation. Oral Surg Oral Med Oral Pathol 1992;74:499-507.

26. Walsch $\mathrm{H}$. The hybrid concept of nickel-titanium rotary instrumentation. Dent Clin North Amer 2004;48:183-202.

27. Gambarini $G$. The $\mathrm{K} 3$ rotary nickel titanium instrument system. Endodontic Topics 2005;10:179-182. 